\title{
Alternative Numerical Techniques
}

\author{
G. S. Guralnik* a , J. Doll ${ }^{\mathrm{a}}$, R. Easther ${ }^{\mathrm{b}}$, P. Emirdag ${ }^{\mathrm{a}}$, D. D. Ferrante ${ }^{\mathrm{a}}$, S. Hahn $^{\mathrm{a}}$, D. Petrov ${ }^{\mathrm{a}}$ and D. \\ $\mathrm{Sabo}^{\mathrm{a}}$.

 \\ ${ }^{\mathrm{b}}$ Columbia University, New York, NY. 10027.
}

Two new approaches to numerical QFT are presented.

\section{Introduction}

"Traditional" Monte Carlo (MC) approaches have produced many good results but they do have well known limitations. These include requiring large amounts of computation time as a result of the necessity to treat fermions very differently from bosons, as well as problems with fermion multiplicity associated with the lattice. Moreover, non-positive definite actions, actions with rapid oscillations including phase transitions and symmetry breaking are generally not tractable. Finally, highly accurate answers can require immense resources.

For these reasons we have been working on two alternative approaches. The first is a tuned Monte Carlo method, which we call Mollified Monte Carlo (MMC), while the second, involving nested approximations to the Schwinger-Dyson equations, is called the Source Galerkin method (SG). These two methods are closely related because MMC is initiated by starting from a stationary phase point of an action, while the simplest applications of SG tend to favor such points in initial iterations. The talks by Ferrante and Petrov [1,2] give a few more details.

\section{Mollified Monte Carlo}

Unlike normal MC, the integrands for problems approached using MMC are very carefully controlled before any actual heavy duty calculation takes place. Regions of high oscillation are identified and smoothed (mollified) by using simple exponential functions that do not change the values of any of the integrals but do suppress unim- portant oscillatory contributions. The resulting integrals are evaluated around stationary points using appropriate importance function weighting and MC integration. In the cases we have examined this approach allows accurate evaluation of normally intractable integrands, including complex integrands. In particular, theories can be directly evaluated in symmetry breaking or other phases with comparative ease. MMC still suffers from the traditional problems associated with fermions (although with the potential for more rapid convergence of integrals) but does considerably extend the class of actions and regions of evaluation open to the usual approaches.

\section{Source Galerkin Methods}

Our Source Galerkin method, while requiring less familiar numerical techniques, is powerful because it is defined on the continuum and treats fermions, except for anti-commutativity, in the same way as bosons. The basic idea is simple: We assume that the QFT action is written with sources $J_{i}(x)$ for every field $\phi_{i}(x)$ so that the amplitude $Z=\langle+0 \mid 0-\rangle_{J_{i}}$ satisfies the differential equation,

$$
\underbrace{F\left(\frac{\delta}{\delta J_{i}(x)}\right)}_{\begin{array}{c}
\text { essentially the } \\
\text { field equations }
\end{array}} z=0 .
$$

A familiar but far from straightforward example is $\phi^{4}$ scalar QFT,

$\left(\partial^{2}+m^{2}\right) \phi(x)+g \phi^{3}(x)=J(x)$, 
which becomes (in Euclidean space),

$\left[\left(\partial_{x}^{2}+m^{2}\right) \frac{\delta}{\delta J(x)}+g\left(\frac{\delta}{\delta J(x)}\right)^{3}-J(x)\right] z[J]=0$.

This is a very non-trivial infinite set of coupled differential equations. To get some idea of its complexity, examine the one point (0-dim) case, $\left[m^{2} \frac{d}{d J}+g\left(\frac{d}{d J}\right)^{3}-J\right] z[J]=0$.

Even this simple equation has three independent solutions and requires the input of parameters for a full solution [3]. These three solutions can be characterized by their degree of singularity:

- Regular at $g \rightarrow 0$ : consistent with perturbation theory;

- $\sim g^{-1 / 2}$ at $g \rightarrow 0$ : "symmetry breaking";

- $\sim \exp \left(\mu^{2} / 4 g\right)$ at $g \rightarrow 0$ : "instanton".

In the path integral language, these solutions correspond to the three saddle points of the path integral in the complex $\phi$-plane.

The Schwinger condition,

$\frac{d z}{d g}=\int(d x)\left(\frac{\delta}{\delta J(x)}\right)^{4} z$

is used to stabilize the phase choice [3] using SG in iterative approximations as is discussed in what follows.

A direct approach to solving these is to assume a truncated functional expansion in the sources and introduce some way to minimize the error. It is the presence of a method for error control that differentiates our approach from any older Schwinger-Dyson approximations. Formally, the correct answer is,

$z=\exp \left\{A_{0}+\int A_{i}(x) J_{i}(x)+\int A_{i j}(x, y) J_{i}(x) J_{j}(y)+\cdots\right\}$

The problem is to introduce a procedure to iteratively calculate the $A_{i}$ 's. In order to do this, we put information we already have about the field theory (spectral information, relativity, LehmanKallen representations) and make decompositions by assuming

$z=\exp \left\{a+\int n J(x)+\int J(x) G(x-y) J(y)\right\}+$

$+\{$ all possible higher order Green's function terms

all constructed from terms involving only functions

$G(x-y)$ but with possibly different parameterization. $\}$
The above representation is highly symbolic. To make calculations tractable we have spanned the solution space involving products of sources by multiplying these, in turn, with all possible products of free field propagators with arbitrary weights and masses. For example, the quartic source contribution, $J J G_{4} J J$ is represented by all possible graphs involving only the "free" $G(x-y)$ with four external lines. The $G(x-y)$ can have arbitrary numerical weights and mass. The result is a very general, very complicated expression consistent with Lorentz and translation invariance.

The propagator $G(x-y)$ is,

$G(x-y)=\int d K^{2} d^{4} k \frac{e^{i k(x-y)}}{-k^{2}+K^{2}} a\left(K^{2}\right)$

This satisfies space-time restrictions, and the $a\left(K^{2}\right)$ are weight functions. When inserted into the field equations the above expansion yields constraints on $a\left(K^{2}\right)$. In general, this is too complicated to directly solve, since this would be tantamount to an exact solution of a field theory. Finally, there are cross-terms in any equation and the equations are non-linear!

We simplify as follows:

- Truncate the expansion in $J$;

- Limit the number of masses in each propagator;

- Limit the number of graphs considered for each $J$.

We do this in an organized, systematic, way so that after a first guess more terms can be included so as to iterate answers.

Roughly this works as follows: Approximating yields $Z^{*}[J]$ and, of course,

$F\left(\frac{\delta}{\delta J}\right) z_{\text {approx }}[J] \neq 0$.

The idea of Source Galerkin is to require that,

$\int d J_{1} \cdots d J_{n} F_{i}\left(J_{1}, \ldots, J_{n}\right) F\left(\frac{\delta}{\delta J}\right) z_{\text {approx }}[J]=0$,

so that $Z_{\text {approx }}[J]$ satisfies the field equations "on the average". An appropriate number of $F_{i}$ are 
picked so that all the undetermined weights in $Z^{*}[J]$ are determined.

In general, these equations are non-linear, and must be solved in a very careful and systematic manner. Theorems for simpler Galerkin approaches promise convergence and we conjecture that the same is true for QFT. We have applied this approach to many models (with amazing accuracy when a check is available) but the stability and convergence must be confirmed. Higher iterations are simple in principle, but introduce computational difficulties. To understand these we have re-examined perturbation theory using variants of our numerical techniques [4] and also reanalyzed trivial 0-dimensional models for higher order Galerkin expansion [2].

\section{Perturbation Theory}

The usual graphical rules apply (not our extended "exact" rules). Application of our ideas have lead to a new way to numerically calculate graphs 细. The major ingredients of our new procedure are the Sinc-function expansion:

$S_{k}(h, x) \equiv \frac{\sin (\pi(x-k h) / h)}{\pi(x-k h) / h}, \quad k \in \mathbb{Z} ;$

and the cutoff propagator

$$
G_{\Lambda}(x)=\int \frac{d^{4} p}{(2 \pi)^{4}} \frac{e^{i p x}}{p^{2}+m^{2}} e^{-p^{2} / \Lambda^{2}} .
$$

Using the Sinc expansion gives [4]

$$
\begin{aligned}
G_{\Lambda, h}(x) & =\frac{m^{2} h}{(4 \pi)^{2}} \sum_{k=-\infty}^{\infty} p(k) \exp \left[-\frac{m^{2} x^{2}}{4 C(k)}\right] \\
C(k) & =e^{k h}+\frac{m^{2}}{\Lambda^{2}}, \quad p(k)=\frac{e^{k h-e^{k h}}}{C^{2}(k)} .
\end{aligned}
$$

Typically, this can be approximated to very high accuracy ( 1 part in $10^{16}$ ) with fewer than 100 terms in the sum and similar statements hold for fermion propagators. Using these propagators, graphs can be reduced to multi-dimensional sums which can then be quickly (relative to the analogous Monte Carlo integral) calculated to high precision. We believe this may be a powerful way to check high order magnetic moment calculations, which are currently of great interest. Technical issues with accuracy using an "auto" renormalization based on approaches used in lattice gauge theory scheme have slowed us down, but we hope to release some more computations emphasizing the power of this method in the near future. Moreover, the ease and speed of these perturbative calculations gives us hope that we can iterate Source Galerkin calculations to moderate order with relatively small amounts of computer time.

\section{Acknowledgments}

DDF, DP and GSG wish to acknowledge support by DOE grant DE-FG02-91ER40688 - Task D, JDD and DS wish to acknowledge support from the National Science Foundation through awards CHE-0095053 and CHE-0131114.

\section{REFERENCES}

1. Mollified Monte Carlo, D.D. Ferrante, J. Doll, G.S. Guralnik, D. Sabo, hep-lat/0209053.

2. A Test of the Source Galerkin Method, D. Petrov, P. Emirdag, G. S. Guralnik, hep-lat/0208024.

3. Theta Vacua and Boundary Conditions of the Schwinger-Dyson Equations, S. Garcia, Z. Guralnik, G. S. Guralnik, hep-th/9612079.

4. Fast Evaluation of Feynman Diagrams, R. Easther, G. S. Guralnik, S. Hahn, Phys. Rev. D61:125001, 2000, hep-ph/9903255.

5. New numerical methods for quantum field theories on the continuum, P. Emirdag, R. Easther, G. S. Guralnik and S. C. Hahn, Nucl. Phys. Proc. Suppl. 83, 938 (2000). 